\title{
A Cooperative Uplink Power Control Scheme for Elastic Data Services in Wireless CDMA Systems
}

\author{
George Alyfantis \\ Communication Networks \\ Laboratory, Department of \\ Informatics \& Telecommunications, \\ University of Athens, \\ Athens 15784, Greece \\ +302107275327 \\ alyf@di.uoa.gr
}

\author{
Stathes Hadjiefthymiades \\ Communication Networks \\ Laboratory, Department of \\ Informatics \& Telecommunications, \\ University of Athens, \\ Athens 15784, Greece \\ +30 2107275425 \\ shadj@di.uoa.gr
}

\author{
Lazaros Merakos \\ Communication Networks \\ Laboratory, Department of \\ Informatics \& Telecommunications, \\ University of Athens, \\ Athens 15784, Greece \\ +302107275323 \\ merakos@di.uoa.gr
}

\begin{abstract}
We consider the uplink power control problem in a single cell CDMA wireless data system. Each user specifies upper and lower QoS bounds. We formulate the considered problem as a game, and first examine the non-cooperative case. We then compare it to its cooperative counterpart (through the Nash bargaining solution). The use of the cooperative scheme shows significant reduction in the transmission power of the mobile terminals, while the achieved QoS is slightly compromised, compared to the noncooperative scheme.
\end{abstract}

\section{Categories and Subject Descriptors}

C.2 [Computer-Communication Networks], C.4 [Performance of Systems], G.1.6 [Numerical Analysis]: Optimization Constrained optimization, Nonlinear Programming

\section{General Terms}

Algorithms, Performance, Design, Theory.

\section{Keywords}

CDMA, power control, Game theory, Nash Bargaining Solution, optimization.

\section{INTRODUCTION}

WCDMA has been widely adopted as the air interface technology for third generation (3G) networks [1]. WCDMA is based on Direct Sequence CDMA (DS-CDMA), which is a spread spectrum technology where user signals are spread over the entire transmission spectrum. Unique digital codes are used to separate the signals from different mobile stations, resulting in simpler statistical multiplexing, without the need for complex time or frequency scheduling.

It is well known that minimizing interference using power control increases capacity [2], [3], [4], [5], and extends battery lifetime. Traditionally, much of the work on power control in CDMA wireless networks was limited to voice traffic sources [2], [3]. However, in recent years, the demand for wireless data services has increased, and significant research effort has been devoted to the efficient management of the wireless resources for bandwidthelastic data services.

A popular approach to the power control problem is based on economic models, as in [6], [7], [8], [9]. Service preferences for each user are represented by a utility function, and game-theoretic methods are applied to study and model the interactions between selfish users. However, equilibrium points of such games (Nash equilibria, [10]) are, typically, not the most efficient operating points. As discussed in [12], the introduction of S-Modular games with pricing resulted in significantly more efficient (but unfair) operating points compared to the Nash equilibrium (NE). Hence, the determination of both efficient and fair operating points in the power control problem remains an issue to be explored.

In this paper, we employ the Nash bargaining solution (NBS) [11] from cooperative game theory, which, by definition, yields Pareto optimal and fair solutions. Close to our work is the arbitration scheme that was proposed in [13]. The authors assumed that users should enjoy equal signal-to-interference ratio (SIR) and maximized the user utility function. The resulting operating point is Pareto optimal and fair [17]. However, the authors examined the problem without taking QoS constraints into account. In this paper, we assume bandwidth elastic, delay intolerant services (e.g., voice, video, and real time file transfers), where the rate may vary, but long delays are not permitted. Specifically, each user imposes individual QoS, SIR-expressed, constraints declared to the base station prior to connection establishment. We focus on the determination of the NBS point, subject to such user QoS constraints.

The rest of the paper is organized as follows. In Section 2, we provide the basic formulations of the non-cooperative power control game. In Section 3, we describe a cooperative power control scheme. Specifically, we formulate the nonlinear problem that corresponds to the NBS, and provide an appropriate optimization algorithm for solving it. Section 4 provides numerical results. Lastly, in Section 5, we provide our conclusions.

\section{POWER CONTROL GAME}

In this section, we formulate the CDMA power control problem as a game. Let $I=\{1, \ldots, N\}$ be the set of users who share the wireless bandwidth of a CDMA cell. User $i$ controls his transmitted power $p_{i}$, which is chosen from set $S_{i}=[0,+\infty)$. Let $\boldsymbol{p}$ $=\left(p_{1}, \ldots, p_{N}\right)^{T}$ be a typical strategy profile vector in the strategy space $S=S_{I} \times \ldots \times S_{N}$.

In CDMA networks, all users transmit, simultaneously, in the same band, thus, interfering with one another. The higher the power with which a user transmits, the better will be his signal 
quality at the base station, but the higher will be the interference to the signals of the other users (and vice versa). The typical metric that quantifies the signal quality of user $i$ is his SIR value $\gamma_{i}$, defined as

$$
\gamma_{i}=\gamma_{i}(\boldsymbol{p})=\frac{W}{R} \frac{h_{i} p_{i}}{\sum_{k \neq i} h_{k} p_{k}+\sigma^{2}},
$$

where $W$ is the chip rate, $R$ the transmission rate, $h_{\mathrm{i}}$ is the path loss from user (mobile terminal) $i$ to the base station, and $\sigma^{2}$ the additive white Gaussian noise (AWGN) power at the receiver. The SIR metric is directly related to the QoS perceived by the user, as it directly influences the bit-error-rate (BER), and, thus, the achieved throughput.

In this paper, we assume that user $i$ has certain QoS requirements expressed by lower and upper bounds on the achieved SIR, $\gamma_{m, i}$ and $\gamma_{M, i}$, respectively $\left(\gamma_{m, i}<\gamma_{M, i}\right)$. Such SIR constraints, define the set of power allocations $\Gamma=\left\{\boldsymbol{p} \in S: \gamma_{m, i} \leq \gamma_{i}(\boldsymbol{p}) \leq \gamma_{M, i}, i \in I\right\}$. It can be easily shown that set $\Gamma$ is a convex polyhedron.

In order to fully describe the power control game, it is also necessary to define the utility functions of the users, i.e., the functions that quantify the level of user satisfaction for using the system resources. The utility function defined in [12] takes into account the achieved QoS as a result of the user's transmitted power and the interference from the other users, as well as the resulting energy consumption. Specifically, it expresses the number of bits that are successfully received at the base station per unit of consumed energy. Let $L$ be the length in bits of a user frame, and $M$ the length of the frame including headers $(M>L)$. Then, for user $i$, the utility function $u_{i}: \Gamma \rightarrow \mathfrak{R}$ is as follows:

$$
u_{i}(\boldsymbol{p})=\frac{L R}{M p_{i}}\left(1-e^{-0.5 \gamma_{i}}\right)^{M}(\text { bits/Joule })
$$

The term $\left(1-e^{-0.5 \gamma_{i}}\right)^{M}$ is an approximation to the probability of correct frame reception for asynchronous FSK modulation, assuming an additive Gaussian channel and no channel coding ${ }^{1}$. More details on the adopted utility function can be found in [12]. Similar versions of the discussed utility function can be found in [15], and [16]. Figure 1 shows the utility function of a user for a given interference level.

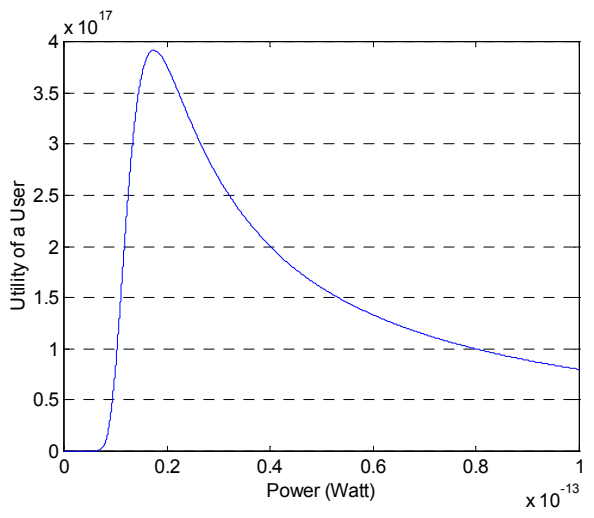

Figure 1. Utility function of a user, for given interference level

\footnotetext{
${ }^{1}$ This simplistic channel model is considered sufficient for modeling the social interaction problem emerging from the power control game [12].
}

\subsection{Nash Equilibrium of the Non-cooperative SIR-constrained Power Control Game}

In this section, based on the formulations introduced, in the previous section, we assume that users act selfishly, i.e., each user, given global system information, tries to selfishly maximize his utility function. The main issue is the existence and uniqueness of a NE, i.e., if, given this selfish user behavior, the system will finally settle to a stable state. The definition of the NE with respect to the discussed problem follows:

Definition 2.1: A power vector $\boldsymbol{p}=\left(p_{1}, \ldots, p_{N}\right)^{\mathrm{T}}$ is a Nash equilibrium of the power control game if for every user $i \in I$,

$$
\begin{gathered}
u_{i}\left(p_{i}, \boldsymbol{p}_{-i}\right) \geq u_{i}\left(p^{\prime}{ }_{i}, \boldsymbol{p}_{-i}\right) \\
\text { for all } p_{i}^{\prime} \in S_{i}, \gamma_{m, i}<\gamma_{i}\left(p^{\prime}{ }_{i}, \boldsymbol{p}_{-i}\right)<\gamma_{M, i},
\end{gathered}
$$

where $\boldsymbol{p}_{-\boldsymbol{i}}=\left(p_{1}, \ldots, p_{i-1}, p_{i+1}, \ldots, p_{N}\right)^{\mathrm{T}}$.

That is, at the NE, given the power levels of the other users, no user can benefit by making individual changes to his transmitted power. In the studied problem, there is one and only one NE, as shown in the following theorem.

Theorem 2.1: There exists a unique Nash equilibrium to the non-cooperative power control game.

Proof: The proof of the above theorem follows from Debreu's Theorem 3.2 in [19], as the utility function given in equation (2) is defined over the convex set (polyhedron) $\Gamma$ and is quasiconcave [14] in $p_{i}$ [12].

In case no SIR constraints are imposed by the users, every user enjoys the same SIR $\gamma^{*}$, at the NE (i.e., $\gamma_{i}^{*}=\gamma_{j}{ }^{*}=\gamma^{*}, \forall i, j \in I$ ). Specifically, the NE SIR $\gamma^{*}$ derives from the first order optimality condition (sufficient in the discussed problem), i.e., $\partial u_{i}(\boldsymbol{p}) / \partial p_{i}=0$, $i \in I$, [12]. It is easy to show that the discussed optimality condition takes the following form:

$$
0.5 M \gamma_{i}+1=e^{0.5 \gamma_{i}}
$$

The value of $\gamma^{*}$ that solves (3) can be readily obtained numerically ${ }^{2}$. However, in case there are SIR constraints, the users do not typically enjoy the same SIR, at the NE. Specifically, the SIR-expressed NE for the SIR-constrained power control game is given as follows:

$$
\gamma_{i}^{*}=\left\{\begin{array}{c}
\gamma_{m, i}, \gamma^{*}<\gamma_{m, i}<\gamma_{M, i} \\
\gamma^{*}, \gamma_{m, i}<\gamma^{*}<\gamma_{M, i} \\
\gamma_{M, i}, \gamma_{m, i}<\gamma_{M, i}<\gamma^{*}
\end{array}\right.
$$

where $\gamma^{*}$ is the solution of equation (3), as already discussed.

The NE SIR of a user, as derives through (3) and (4), can be easily calculated by the user; it only depends upon the characteristics of the specific user (e.g., SIR bounds of the user). However, the power to achieve the NE SIR, results through the interaction among users, and, thus, depends on their collective

\footnotetext{
${ }^{2}$ Note that $\gamma_{i}=0$ solves (3). However, at that point, the utility function $u_{i}($ ) assumes a zero value, which implies that $\gamma^{*} \neq 0$.
} 
characteristics. To clarify this we should note that, in distributed power control schemes, the transmitted power of user $i$ converges to the NE power $p_{i}{ }^{*}$ (where the target SIR $\gamma_{i}{ }^{*}$ is achieved) by means of the following iterative procedure.

$$
p_{i}(t+1)=\frac{R \gamma_{i}^{*}}{W h_{i}}\left[r p(t)-h_{i} p_{i}(t)\right],
$$

where $r p(t)$ is the total received power at the base station at timeslot $t$, i.e.,

$$
r p(t)=\sum_{j=1}^{N} h_{j} p_{j}(t)
$$

Such information is regularly broadcast by the base station. If $p_{i}(t+1)$ exceeds the maximum transmission power of the user terminal, $p_{\max }$, then $p_{i}(t+1)$ is reduced back to $p_{\max }$.

It is easy to see that the iterative procedure introduced in (5) is the distributed solution to the linear system of equations that derives from (1), when solving for the transmitted powers of the users, given their target, NE SIRs. This procedure allows solving the problem without assuming that users know all system parameters (i.e., each user needs only know the total received power at the base station $r p(t)$, and his target $\operatorname{SIR} \gamma_{i}^{*}$ ).

By analytically solving the linear system of SIR equations, we obtain the following expression of power $q_{i}{ }^{*}=h_{i} p_{i}{ }^{*}$ with which user $i$ will reach the base station:

$$
q_{i}^{*}=\sigma^{2}\left\{\left(\frac{W}{R \gamma_{i}^{*}}+1\right)\left(1-\sum_{k=1}^{N}\left(\frac{W}{R \gamma_{k}^{*}}+1\right)^{-1}\right)\right\}^{-1}
$$

From (6), we observe that for feasible (nonnegative and finite) power allocations the following condition must hold:

$$
\sum_{k=1}^{N}\left(\frac{W}{R \gamma_{k}^{*}}+1\right)^{-1}<1
$$

If the condition in (7) does not hold true, the iterative procedure in (5) will not converge. Specifically, in the absence of the maximum power constraint, $p_{\max }$, the transmitted power of users will become infinite. For the case of bounded transmitted powers, all users will transmit at maximum power, without, however achieving their target, NE SIR. Hence, it is not possible that all users be admitted in the network and at the same time be capable of achieving their target SIRs. A mechanism for granting or denying network connection to users is required.

We assume that a new user declares his SIR requirements (i.e., $\left.\gamma_{i}^{*}\right)$ to the base station before attaching. Then the base station applies a simple connection admission control (CAC) procedure checking whether (7) is violated by taking into account the contribution of the new user. The new user is allowed to attach to the base station only if (7) holds true.

Another important issue is the efficiency of the NE. A common indicator of efficiency in multi-objective optimization problems (e.g., games) is Pareto optimality (or efficiency). A formal definition of Pareto optimality follows.
Definition 2.3: The point $\boldsymbol{u} \in U$, where $U$ is the set of achievable utilities, is said to be Pareto optimal if for each $v \in U$, $\boldsymbol{v} \geq \boldsymbol{u}$, then $\boldsymbol{v}=\boldsymbol{u}$.

In other words, it is impossible to find another point than the Pareto optimum, which yields strictly superior utility for all users simultaneously. In the unconstrained, non-cooperative power control game considered in [12], the authors proved that the NE is Pareto inefficient. Quite similarly, the inefficiency of the NE of the SIR-constrained version of the power control game can also be proved. Moreover, as will be shown in Section 4, the inefficiency of the NE discussed here is evident.

\section{ARBITRATED SIR-CONSTRAINED POWER CONTROL}

In this section, we investigate the application of the NBS in the considered power control game. In this setting, users are not allowed to act selfishly. By being enforced to cooperate by the arbitration mechanism, Pareto optimal and fair results are achieved.

\subsection{The Nash Bargaining Solution}

In the context of cooperative game theory, user $i$, apart from his utility function $u_{i}$, has also a desired initial utility $u_{i}^{0}$, which corresponds to the minimum utility that he can achieve without cooperation (status quo utility). An arbitration mechanism must always provide utility that is superior to the status quo utility for every user. Otherwise, the user has no incentive to cooperate. Here, $u_{i}^{0}=u_{i}\left(\boldsymbol{p}^{*}\right)$, where $\boldsymbol{p}^{*}$ is the NE power vector. We will refer to $\boldsymbol{u}^{\boldsymbol{\theta}}=\left(u_{1}^{0}, \ldots, u_{N}{ }^{0}\right)$ as the status quo of the game.

A formal definition of the NBS follows.

Definition 3.1: A mapping $F: G \rightarrow \mathfrak{R}^{N}$, where $G$ denotes the set of achievable utilities with respect to the status quo $\boldsymbol{u}^{0}$, is said to be a NBS, if the following hold:

1. $F\left(U, \boldsymbol{u}^{0}\right) \in U_{0}$, where $U_{0}$ is the set of achievable utilities that are superior to the status quo utility.

2. $F\left(U, \boldsymbol{u}^{0}\right)$ is Pareto optimal.

3. $F$ satisfies the linearity axiom: if $\boldsymbol{\varphi}: \mathfrak{R}^{N} \rightarrow \mathfrak{R}^{N}, \boldsymbol{\varphi}(\boldsymbol{u})=\boldsymbol{u}^{\prime}$ with $u_{j}^{\prime}=a_{j} u_{j}+b_{j}, a_{j}>0, j=1, \ldots, N$, then $F\left(\varphi(\boldsymbol{u}), \boldsymbol{\varphi}\left(\boldsymbol{u}^{0}\right)\right)=\boldsymbol{\varphi}(F(\boldsymbol{u}$,

4. $F$ satisfies the irrelevant alternatives axiom: if $V \subset U,\left(V, \boldsymbol{u}^{0}\right)$ $\in G$ and $F\left(U, \boldsymbol{u}^{0}\right) \in V$, then $F\left(U, \boldsymbol{u}^{0}\right)=F\left(V, \boldsymbol{u}^{0}\right)$.

5. $F$ satisfies the symmetry axiom: if $U$ is symmetric with respect to a subset $J \subseteq\{1, \ldots, N\}$ of indices (i.e., $\boldsymbol{u} \in U$ and $i, j \in J$, then if $u_{i}^{0}=u_{j}^{0}$ then $F\left(U, \boldsymbol{u}^{0}\right)_{i}=F\left(U, \boldsymbol{u}^{0}\right)_{j}$ for $i, j \in J)$.

The first and second items are the known axioms regarding the superiority of the solution to the status quo and the requirement for Pareto optimality, respectively. Items 3, 4, and 5 are often referred to as axioms of fairness. The linearity axiom (3) of the solution implies that the bargaining solution is scale invariant, i.e., it is not affected by affine transformations of the utility functions. The irrelevant-alternatives axiom (4) implies that the bargaining solution point is not affected by extending the domain of utilities, if agreement can be found on a restricted domain. The symmetry axiom (5) states that the bargaining solution point is irrelevant to 
specific user labels, i.e., users with the same status quo and utility function will always achieve the same outcome.

The solution of Nash, which satisfies all of the above axioms, is achieved at the point where the product of the utility functions of the users, with respect to the status quo of the game is maximized $^{3}$. For the problem considered in this paper, the NBS point of the power control game is the solution of the optimization problem $(P)$.

$$
\left\{\begin{array}{l}
\max _{\boldsymbol{p}} f(\boldsymbol{p}), f(\boldsymbol{p})=\prod_{j=1}^{N}\left(u_{j}(\boldsymbol{p})-u_{j}^{0}\right) \\
\boldsymbol{p} \in X, X=\left\{\boldsymbol{r} \in \Gamma: \boldsymbol{u}(\boldsymbol{r})>\boldsymbol{u}^{0}\right\}
\end{array}\right.
$$

In other words, at the NBS point, the product of utilities of the involved users is maximized, subject to the constraint that the SIR of every user must be within the respective bounds and that the utility of each user must be superior to his status quo utility.

In order to solve $(P)$, we need to study whether function $f(\cdot)$ has a maximum, and whether this is unique. From the quasiconcavity of function $f(\cdot)$ over the convex set $X$ [18], it follows that the stationary point of the objective function is the global maximum. Hence, the optimization problem $(P)$ has a unique solution.

Let us now define optimization problem $\left(P^{\prime}\right)$, which is equivalent to the problem $(P)$, but less complex, as the objective function becomes a sum of functions, rather than a product [22]. In $\left(P^{\prime}\right)$, the objective function is the logarithm of the objective function $f()$ of $(P)$.

$$
\left(P^{\prime}\right)\left\{\begin{array}{l}
\max _{\boldsymbol{p}} \sum_{j=1}^{N} \ln \left(u_{j}(\boldsymbol{p})-u_{j}^{0}\right), \\
\boldsymbol{p} \in X, X=\left\{\boldsymbol{r} \in \Gamma: \boldsymbol{u}(\boldsymbol{r})>\boldsymbol{u}^{0}\right\}
\end{array}\right.
$$

As the function composition with the logarithm function, $\ln : \mathfrak{R}_{+} \rightarrow$ $\mathfrak{R}$, is an operation that preserves quasiconcavity, the objective function in problem $\left(P^{\prime}\right)$ is quasiconcave. Hence, it can be readily shown that, if problem $(P)$ has a global maximum $\widetilde{\boldsymbol{p}}$, then $\left(P^{\prime}\right)$ will have the same global maximum $\tilde{\boldsymbol{p}}$ [22]. We, finally, formulate the optimization problem $\left(P^{\prime \prime}\right)$, which can be easily shown to be equivalent to $\left(P^{\prime}\right)$.

$$
\left(P^{\prime \prime}\right)\left\{\begin{array}{l}
\max _{\boldsymbol{q}} g(\boldsymbol{q}), g(\boldsymbol{q})=\sum_{j=1}^{N} \ln \left(v_{j}(\boldsymbol{q})-v_{j}^{0}\right), \\
\boldsymbol{q} \in X, X=\left\{\boldsymbol{r} \in \Gamma: \boldsymbol{v}(\boldsymbol{r})>\boldsymbol{v}^{0}\right\}
\end{array}\right.
$$

where $v_{j}(\boldsymbol{q})=h_{j} u_{j}(\boldsymbol{p})$ and $v_{j}^{0}=h_{j} u_{j}^{0}$. In $\left(P^{\prime \prime}\right)$ we need to solve for the received power vector $\boldsymbol{q}$, regardless of the path losses, which is more convenient for the centralized control scheme discussed here.

\footnotetext{
3 This is quite similar to proportional fairness, often encountered in networking problems, such as flow control, where a number of flows have to pass through a system of finite capacity, and the objective is to share this capacity in a fair manner [21].
}

\subsection{Centralized Algorithm for SIR- constrained Power Control}

In this section, we discuss the iterative algorithm executed by the CDMA base station for solving $\left(P^{\prime \prime}\right)$ (i.e., derive the NBS point of the game). Such algorithm is executed every time a new user attaches to the system, or when an active user disconnects. After such events, the updated optimal received power vector $\tilde{\boldsymbol{q}}$ is broadcast so that every attached user $i$ adjusts his transmission power $\tilde{p}_{i}=\tilde{q}_{i} / h_{i}$ accordingly. We assume that when $\tilde{q}_{i}$ cannot be reached, due to the maximum transmitted power limitation, the user may disconnect in case his minimum SIR constraint, $\gamma_{m, i}$, is not satisfied.

The proposed algorithm is based on the conditional gradient method [20]. We shall not discuss the details of the algorithm, but will provide an overview of its basic components: (1) determination of feasible ascent directions, (2) stepsize selection, and (3) initial point selection.

\subsubsection{Feasible Direction Finding Subproblem}

A feasible direction method starts with a feasible vector $\boldsymbol{q}(0)$ and generates a sequence of feasible vectors $\{\boldsymbol{q}(n)\}$ according to

$$
\boldsymbol{q}(n+1)=\boldsymbol{q}(n)+a(n) \boldsymbol{d}(n),
$$

where $\boldsymbol{d}(n)$ is a feasible direction at $\boldsymbol{q}(n)$, and also an ascent direction, i.e., $\nabla g(\boldsymbol{q}(n))^{\mathrm{T}} \cdot \boldsymbol{d}(n)>0$. The stepsize is chosen to be positive and such that $\boldsymbol{q}(n+1) \in X$, and

$$
g(\boldsymbol{q}(n)+a(n) \boldsymbol{d}(n))>g(\boldsymbol{q}(n)), \forall n \in\{0,1, \ldots\} .
$$

In the case, where $X$ is convex (like in the studied problem), the feasible direction method can be written in the form

$$
\boldsymbol{q}(n+1)=\boldsymbol{q}(n)+a(n)(\overline{\boldsymbol{q}}(n)-\boldsymbol{q}(n)),
$$

where $\overline{\boldsymbol{q}}(n)$ is some feasible vector, and $a(n) \in(0,1]$, and if $\boldsymbol{q}(n)$ is non stationary (i.e., $\forall g(\boldsymbol{q}(n)) \neq 0$ ),

$$
\overline{\boldsymbol{q}}(n) \in X, \quad \nabla g(\boldsymbol{q}(n))^{T} \cdot(\overline{\boldsymbol{q}}(n)-\boldsymbol{q}(n))>0 .
$$

We now describe how we choose a feasible direction given a feasible point $\boldsymbol{q}(n)$. The most straightforward way to generate a feasible direction $\overline{\boldsymbol{q}}(n)-\boldsymbol{q}(n)$ that satisfies the ascent condition $\nabla g(\boldsymbol{q}(n))^{T} \cdot(\overline{\boldsymbol{q}}(n)-\boldsymbol{q}(n))>0$ is as follows:

$$
\overline{\boldsymbol{q}}(n)=\underset{\boldsymbol{w} \in \boldsymbol{X}}{\arg \max } \nabla g(\boldsymbol{q}(n))^{T} \cdot(\boldsymbol{w}-\boldsymbol{q}(n)) .
$$

That is to say, point $\overline{\boldsymbol{q}}(n)$ is the remotest point of $X$ along the gradient direction.

Such approach is usually adopted when $g(\cdot)$ is nonlinear, whereas the constraint set $X$ comprises linear constraints. In such case, we have a linear program that is easy to solve. However, in our case, the constraint set includes nonlinear constraints (i.e., each user must receive strictly superior utility to his status quo utility ). For this reason, we propose a two-phase direction finding procedure. Firstly, we only consider the linear constraints, i.e., the SIR constraints that form the convex polyhedron $\Gamma$. Secondly, we consider the nonlinear constraints that stem from the fact that users must achieve higher utility compared to their status quo utility. 
Figure 2 outlines the two-phase direction finding procedure, in a two-dimensional space. In Figure 2(a), only the linear constraints are taken into account. However, the resulting point $\overline{\boldsymbol{q}}(n)$ need not necessarily be feasible, as shown in Figure 2(b). It may lie outside the set $Q_{0}=\left\{\boldsymbol{q} \in S \mid \boldsymbol{v}(\boldsymbol{q})>\boldsymbol{v}^{0}\right\}$, which implies that at least one user receives inferior utility to his status quo utility.

Note that since the set $Q_{0}$ is convex ${ }^{4}$, it is possible to locate a point $\overline{\boldsymbol{q}}^{\prime}(n)$ on the line connecting points $\boldsymbol{q}(n)$ and $\overline{\boldsymbol{q}}(n)$, such that $\overline{\boldsymbol{q}}^{\prime}(n) \in Q_{0}$. (see Figure 2(c)). Hence, the second phase of the direction finding procedure is to locate the point $\overline{\boldsymbol{q}}^{\prime}(n)$; the direction $\boldsymbol{d}(n)$ at step $n$ would then be $\boldsymbol{d}(n)=\overline{\boldsymbol{q}}^{\prime}(n)-\boldsymbol{q}(n)$.
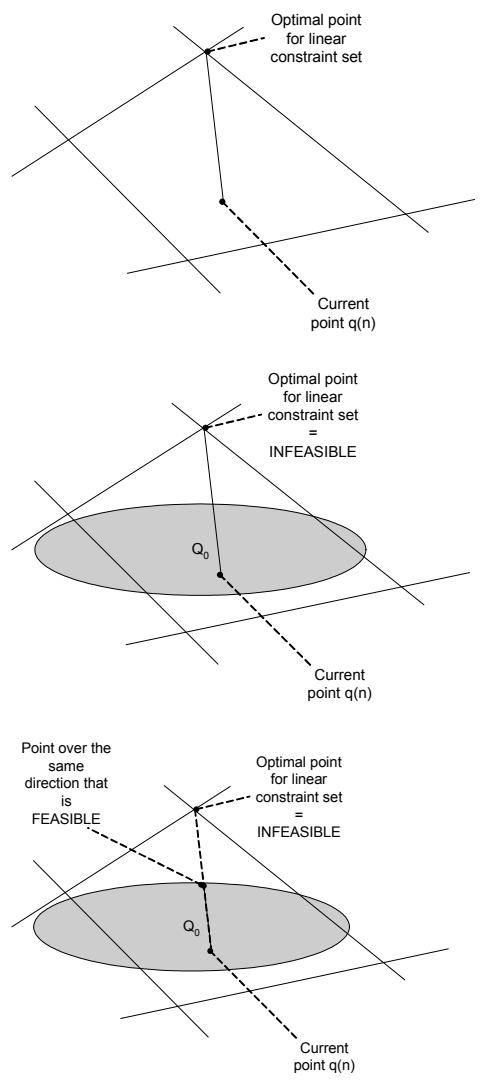

Figure 2. Two-Phase direction selection procedure. (a) Optimal direction subject to linear constraints only, (b) The optimal direction subject to the linear constraints is not feasible, (c) the vector pointing to the initial direction is readjusted to fall within the feasible region.

Below, we summarize the feasible direction finding algorithm.

\footnotetext{
${ }^{4}$ This is due to the quasiconcavity of the utility function $v_{i}()$ - every super-level set of function $v_{i}\left({ }^{\prime}\right)$ is convex, thus, $Q_{0}=\bigcap_{j=1}^{N}\left\{\boldsymbol{q} \in S \mid \boldsymbol{v}(\boldsymbol{q})>\boldsymbol{v}^{0}\right\}$ is convex.
}

1. Calculate the gradient $\nabla g(\cdot)$ of the objective function $g(\cdot)$ at point $\boldsymbol{q}(n)$

2. Solve the linear problem to determine the point $\overline{\boldsymbol{q}}(n)$ that maximizes the inner product $\nabla g(\boldsymbol{q}(n))^{\mathrm{T}} \cdot \boldsymbol{q}$ over the polyhedron $\Gamma$

3. If $\overline{\boldsymbol{q}}(n)$ is feasible (i.e., $\overline{\boldsymbol{q}}(n) \in Q_{0}$ - all users enjoy higher utility compared to the status quo), then go to step 8 . Else go to step 4

4. Set $\boldsymbol{l} \_\boldsymbol{q}:=\boldsymbol{q}(n), \boldsymbol{r} \_\boldsymbol{q}:=\overline{\boldsymbol{q}}(n)$, and $\boldsymbol{q}:=0.5 \cdot\left(\boldsymbol{r} \_\boldsymbol{q}+\boldsymbol{l} \_\boldsymbol{q}\right)$

5. If $\boldsymbol{q} \in Q_{0}$ then set $\boldsymbol{l}_{-} \boldsymbol{q}:=\boldsymbol{q}$. Else set $\boldsymbol{r}_{-} \boldsymbol{q}:=\boldsymbol{q}$.

6. Set $\boldsymbol{q} \_$old $:=\boldsymbol{q}$ and $\boldsymbol{q}:=0.5 \cdot\left(\boldsymbol{r} \_\boldsymbol{q}+\boldsymbol{l} \_\boldsymbol{q}\right)$

7. If $\| \boldsymbol{q} \_$old $-\boldsymbol{q} \|_{2}<\varepsilon$, then $\overline{\boldsymbol{q}}(n):=\boldsymbol{q}$, and go to step 8. Else go to step 5 .

8. $\quad \boldsymbol{d}(n):=\overline{\boldsymbol{q}}(n)-\boldsymbol{q}(n)$. Finish

The constant $\varepsilon$ in step 7 is a sufficiently small positive scalar, used for the algorithm termination. Phase one comprises steps 13; phase two covers the steps 4-7.

\subsubsection{Stepsize Selection}

In iterative optimization algorithms, apart from the problem of finding feasible ascent directions, the selection of the stepsize is also critical for the speed of convergence, as well as for the convergence per se. In this paper, we propose a stepsize selection method based on the Armijo Rule [20]. Every limit point of the conditional gradient method with the Armijo stepsize selection rule is stationary [20]. For a direction $\boldsymbol{d}(n)$ such that $\boldsymbol{q}(n)+\boldsymbol{d}(n)$ is feasible, let $\beta$ and $s$ be fixed scalars, with $\beta \in(0,1)$, and $s \in(0,1)$. We set $a(n)=\beta^{m(n)}$, where $m(n)$ is the smallest integer $m$ such that

$$
g\left(\boldsymbol{q}(n)+\beta^{m} \boldsymbol{d}(n)\right)-g(\boldsymbol{q}(n)) \geq s \beta^{m} \nabla g(\boldsymbol{q}(n))^{T} \cdot \boldsymbol{d}(n)
$$

In other words, the stepsizes $\beta, \beta^{2}, \ldots$, are tried successively, until the above inequality is satisfied for $m=m(n)$. The stepsize $a(n)$ at iteration $n$ is chosen not only to produce a positive improvement in the objective function; as per the test (8), such improvement shall be sufficiently large. In our simulations, we set $\beta=0.1$ and $s$ $=0.2$.

\subsubsection{Determination of an Initial Feasible Point}

In order to apply the conditional gradient method we need to have an initial feasible point $\boldsymbol{q}(0)$. It is observed that being at the NE, by simultaneously reducing each coordinate of the power vector, we may achieve a Pareto improvement, i.e., obtain a power vector that belongs to $Q_{0}$. Hence, we may state that the vector $(-1, \ldots,-$ $1)^{\mathrm{T}}$ is a direction of ascent for the objective function $g(\cdot)$ at the NE $q^{*}$. Moreover, if the discounted power vector lies inside the polyhedron $\Gamma$, such vector is feasible. Hence, by interpreting the direction $(-1, \ldots,-1)^{\mathrm{T}}$ as the gradient of the objective function ${ }^{5}$, we may provide a feasible direction $\boldsymbol{d}$ originating at point $\boldsymbol{q}^{*}$ (see Section 3.2.1). Then, by using the Armijo rule (see Section 3.2.2), we may find a suitable step $a$ for the pair $\left(q^{*}, d\right)$, thus, determining the initial feasible point $\boldsymbol{q}(0)=\boldsymbol{q}^{*}+a \boldsymbol{d}$.

\footnotetext{
${ }^{5}$ Note that the gradient of the objective function is not defined at the status quo of the game.
} 


\subsubsection{Discussion}

The advantage of the algorithm discussed above is that it does not rely on any structure of the constraint set other than its convexity, and the generated sequence of feasible points derives simply by searching along ascent directions. As shown in [20], the direction sequence $\{\boldsymbol{d}(n)\}$ produced by the conditional gradient method is gradient related and converges to a stationary point - here the global maximum of the objective function $g(\cdot)$. Specifically, it is proven that every limit point of the conditional gradient method with the Armijo stepsize selection rule is stationary.

With regards to the speed of convergence of the conditional gradient method, if the constraint set is a polyhedron, the asymptotic rate of convergence of the method is not very fast [20]. However, as the total number of constraints increases, the performance of the method improves [20]. In the studied problem, we have $2 N$ linear (SIR) constraints, as well as $N$ nonlinear (utility) constraints. As a result, the observed convergence rate was quite fast.

\subsection{Enforcement of the NBS Operating Point}

In this section, we discuss how the centrally computed NBS point can be enforced to users that are by definition selfish, and may have the incentive to deviate. Contrary to the NE, at which no user can benefit by changing his strategy unilaterally, i.e., constitutes a stable operating point, the NBS point is not stable as will be explained below.

As reported in [16], if user $i$ transmits with a power that yields an increase of $a \mathrm{~W}$ to his power at the base station, compared to his NBS power $\widetilde{q}_{i}$ (i.e., $\widetilde{q}_{i}+a$ ), then the user's SIR $\gamma_{i}$ will increase by

$$
\Delta \gamma_{i}=\frac{W}{R} \frac{a}{\sum_{k \neq i} \tilde{q}_{k}+\sigma^{2}} .
$$

At the same time, the SIR of the other users will deteriorate. Hence, the system ought to "punish" non-cooperating users so as to secure stability. As proposed in [16], the base station, upon detecting that user $i$ exceeds his NBS power $\tilde{q}_{i}$, may increase intentionally the BER of that user (by flipping the received user bits with a probability that is proportional to the extent of user disobedience), thus, imposing an upper bound on his achieved throughput. As a result, the user cannot benefit by increasing his power, since this would imply increased energy consumption with no BER improvement [16].

\subsection{Connection Admission Control}

In this section, we have studied the arbitrated power control game. As already discussed, it is anticipated that, at the NBS point, users will transmit with decreased power, compared to the NE, thus, their SIR will be decreased as well. As a result, more users can be, theoretically, admitted, based on the test in (7), compared to the non-cooperative setting. However, as will be discussed below, such SIR decrease cannot be exploited in order to increase the number of admitted users.

The NBS is inherently linked to the non-cooperative game and the respective NE. Specifically, every user involved in the game is required to enjoy a utility that is superior to his NE (status quo) utility. The notion of the status quo utility is, thus, central in the NBS. As a result, a user that cannot be considered, in the non- cooperative game, cannot be considered in the NBS either. For example, if load exceeds system capacity (i.e., (7) does not hold true for the NE SIRs), the status quo utilities of the users cannot be defined. Hence, problem $\left(P^{\prime \prime}\right)$ that has the vector of status quo utilities as a reference point cannot be solved. In other words, the NBS may yield operating points with decreased SIR, however, the dependence of the NBS to the status quo confines us to finally consider the NE SIRs in (7), when taking CAC decisions.

\section{NUMERICAL RESULTS}

In this section, we provide results of the performance assessment of the proposed power control scheme. As reported in [12], the unconstrained, non-cooperative power control scheme, with the parameters of Table 1 , achieves a NE SIR $\gamma^{*} \approx 12.42$ (see also equation (3)), and can support up to nine users (see equation (7)). In order to keep the work in [12] as a reference point to the proposed scheme, our study also involves a population of nine users. Without loss of generality, we assume that these nine users have the following SIR-expressed QoS requirements: $\gamma_{\boldsymbol{m}}=(2,4$, $8,5,6,5,3,12,14)$, and $\gamma_{M}=(10,15,20,14,10,22,18,15,17)$.

Table 1. The list of parameters for the single-cell CDMA system

\begin{tabular}{|l|l|}
\hline $\boldsymbol{M}$, total number of bits per frame & 80 \\
\hline $\boldsymbol{L}$, number of information bits per frame & 64 \\
\hline $\boldsymbol{W}$, spread spectrum bandwidth & $10^{6} \mathrm{~Hz}$ \\
\hline $\boldsymbol{R}$, bit rate & $10^{4} \mathrm{~b} / \mathrm{s}$ \\
\hline $\boldsymbol{\sigma}^{2}$, AWGN power at the receiver & $5 \times 10^{-15} \mathrm{~W}$ \\
\hline Modulation technique & non coherent FSK \\
\hline $\boldsymbol{p}_{\boldsymbol{m a x}}$, maximum power constraint & $2 \mathrm{~W}$ \\
\hline
\end{tabular}

Figure 3 shows the utility achieved for user $i$ at the NE and NBS operating points, regardless of the associated path loss, $h_{i}$; i.e., Figure 3 plots $v_{i}(\tilde{\boldsymbol{q}})=h_{i} u_{i}(\tilde{\boldsymbol{p}})$, for each $i=1, \ldots, 9$. Owing to the Pareto optimality of the NBS, the anticipated utility improvement with regards to the NE is significant.

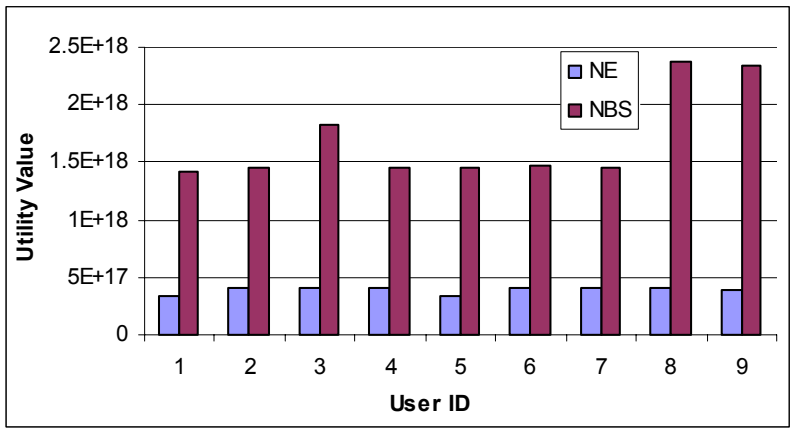

Figure 3. Utility level in the non-cooperative and cooperative schemes

In Figure 4, we compare the received power $q_{i}$ from user $i$ at the base station for the non-cooperative and the cooperative scheme, $q_{i}{ }^{*}$ and $\widetilde{q}_{i}$, respectively. Observe that the NBS operating point is characterized by significant energy savings, compared to the selfish, non-cooperative operation.

In Figure 5, we observe that, at the NBS operating point, the SIR is inferior to the NE SIR. However, such deterioration is rather minor compared to the achieved energy savings, as reflected in the utility values shown in Figure 3. 


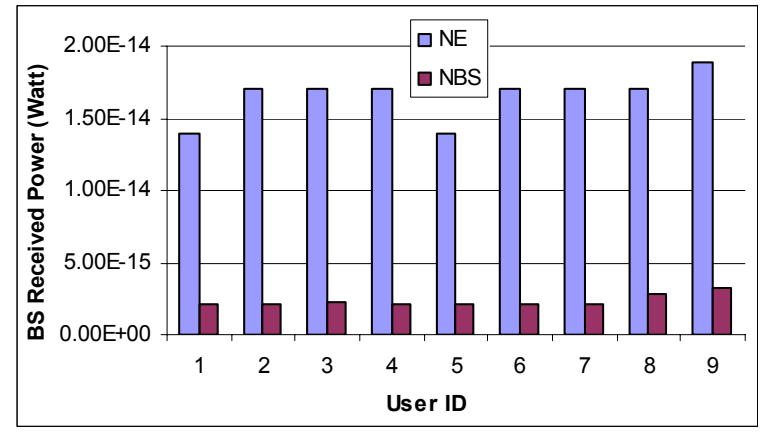

Figure 4. Received power for the non-cooperative and cooperative schemes.

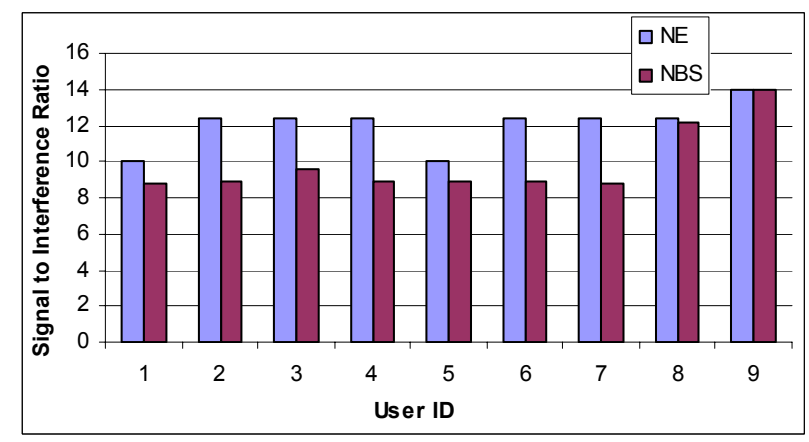

Figure 5. Signal-to-interference ratio (SIR) for the noncooperative and cooperative schemes.

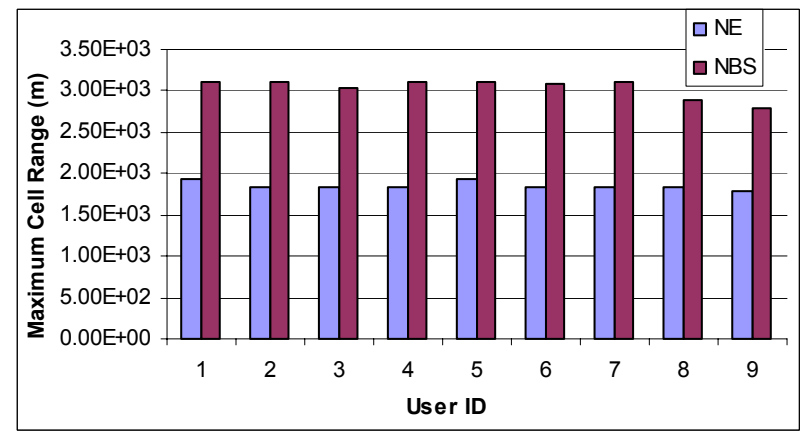

Figure 6. Maximum cell radius for the non-cooperative and cooperative schemes.

We now study the effect of employing the non-cooperative or cooperative scheme at maximum user distance, subject to the maximum transmitted power constraint. For user $i$ we assume the simple path loss model $h_{i}=K_{l} / d_{i}^{4}$, where $K_{l}=0.097$, as in [15], and $d_{i}$ denotes the distance between the user and the base station. Given the maximum transmission power $p_{\max }$ (see Table 1), the maximum distance $d_{i, \max }$ from which user $i$ is capable of reaching the base station is as follows:

$$
d_{i, \max }=\sqrt[4]{\frac{K_{1} p_{\max }}{q_{i}}},
$$

where $q_{i}$ is equal to $q_{i}^{*}$ or $\widetilde{q}_{i}$ for the NE or the NBS operating point, respectively. Figure 6 shows that at the NBS operating point a considerable increase in the maximum cell range is achieved, compared to the NE.

\section{CONCLUSION}

In this paper, we have proposed a centralized power control algorithm for CDMA wireless data networks, subject to SIR constraints imposed by users. Specifically, we have employed the Nash bargaining solution and achieved a Pareto optimal and fair outcome. Despite the overhead that a centralized algorithm entails to the base station for the determination of the NBS operating points, we found that the anticipated Pareto improvement offered by the cooperative scheme, compared to the non-cooperative scheme, is significant. Specifically, the operating points of the proposed cooperative scheme are characterized by significantly reduced transmitted power, and a rather limited decrease of the signal to interference ratio, thus, leading to an increase in the lifetime of the user terminal battery. Furthermore, an increase in the maximum range of the cell has also been observed.

In the future, we plan to extend our study to multi-cell environments. Moreover, we would like to study decentralized mechanisms for enforcing the NBS point, in a distributed manner, e.g., through pricing of wireless resources.

\section{ACKNOWLEDGEMENTS}

The authors would like to thank the anonymous reviewers for their constructive comments, which helped in greatly improving the paper. The first author acknowledges the financial support of Alexander S. Onassis Public Benefit Foundation.

\section{REFERENCES}

[1] H. Holma and A. Toskala, "WCDMA for UMTS", Wiley, New York, 2002.

[2] R. D. Yates, “A framework for uplink power control in cellular radio systems", IEEE JSAC, 13(7), pp. 1341-1347, 1995.

[3] J. Zander, "Performance of optimum transmitter power control in cellular radio systems", IEEE Transactions on Vehicular Technology, 41(1), pp. 57-62, 1992.

[4] S. Grandhi, R. Yates and D. J. Goodman, "Resource allocation for cellular radio systems", IEEE Transactions on Vehicular Technology, 46(3), pp. 581-587, 1997.

[5] D. M. Novakovic and M. L. Dukic, "Evolution of the Power Control Techniques for DS-CDMA Toward 3G Wireless Communication Systems", IEEE Communications Surveys and Tutorials 3(4), 2000.

[6] V. Shah, N. B. Mandayam and D. J. Goodman, "Power control for wireless data based on utility and pricing", Proc. PIMRC, September 1998.

[7] D. Famolari, N. B. Mandayam and D. J. Goodman, "A new framework for power control in wireless data networks: Games, utility and pricing”, Proc. Allerton Conference, September 1998.

[8] N. Feng, N. B. Mandayam and D. J. Goodman, "Joint power and rate optimization for wireless data services based on utility functions", Proc. CISS, March 1999.

[9] H. Ji and C. Huang, "Non-cooperative uplink power control in cellular radio systems", Wireless Networks, (4), pp.233240, 1998. 
[10] D. Fudenberg and J. Tirole, "Game Theory", MIT Press, Cambridge (MA), 1991.

[11] J. F. Nash, "The Bargaining Problem", Econometrica, 18, pp. 155-162, 1950.

[12] C. U. Saraydar, N. B. Mandayam and D. J. Goodman, "Efficient power control via pricing in wireless data networks", IEEE Transactions on Communications, Vol. 50, pp. 291-303, 2002.

[13] D. J. Goodman and N. B. Mandayam, "Network Assisted Power Control for Wireless Data", Kluwer Mobile Networks and Applications 6, pp. 409-415, 2001.

[14] A.W. Roberts and D.E. Varberg, "Convex Functions", Academic Press, Inc., 1973.

[15] S. Gunturi and F. Paganini, "Game theoretic approach to power control in cellular CDMA", Proc. IEEE Vehicular Technology Conference, Orlando, October2003.

[16] A. B. MacKenzie and S. Wiker. "Game Theory in Communications: Motivation, explanation and application to power control”, Proc. IEEE Globecom, November 2001.
[17] G. Alyfantis, S. Hadjiefthymiades and L. Merakos, "Fair and Efficient Power Control in Wireless Data Networks", CNLUoA Technical Report, AL-0505, Communications Network Laboratory, University of Athens.

[18] G. Alyfantis, S. Hadjiefthymiades and L. Merakos, "Optimal and Fair SIR-constrained Uplink Power Control in CDMA Networks", CNL-UoA Technical Report, AL-0805, Communications Network Laboratory, University of Athens.

[19] G. Debreu, "A Social Equilibrium Existence Theorem", in Proceedings of the National Academy of Sciences, Vol. 38, pp 886-893, 1952.

[20] D. P. Bertsekas, "Nonlinear Programming", Belmont, MA: Athena Scientific, 1995.

[21] C. Courcoubetis and R. Weber, "Pricing Telecommunication Networks", Wiley, 2003

[22] H. Yaiche, R. M. Mazumdar and C. Rosenberg, "A Game Theoretic Framework for Bandwidth Allocation and Pricing in Broadband Networks", IEEE/ACM Transactions on Networking, vol. 8, pp. 667-678, 2000. 